K. Sakai

Nagoya Math. J.

Vol. 126 (1992), 125-140

\title{
DIFFEOMORPHISMS WITH PSEUDO ORBIT TRACING PROPERTY
}

\author{
KAZUHIRO SAKAI
}

We shall discuss a differentiable invariant that arises when we consider a class of diffeomorphisms having the pseudo orbit tracing property (abbrev. POTP ) .

Let $M$ be a closed $C^{\infty}$ manifold and $\operatorname{Diff}^{1}(M)$ be the space of diffeomorphisms of $M$ endowed with the $C^{1}$ topology. We denote $\mathscr{P}^{1}(M)$ the $C^{1}$ interior of the set of all diffeomorphisms having POTP belonging to $\operatorname{Diff}^{1}(M)$. Recently Aoki [1] proved that the $C^{1}$ interior of the set of all diffeomorphisms whose periodic points are hyperboric, $\mathscr{F}^{1}(M)$, is characterized as Axiom $A$ diffeomorphisms with nocycle. After this Moriyasu [8] showed that $\mathscr{P}^{1}(M) \subset \mathscr{F}^{1}(M)$ and if $\operatorname{dim} M=2$ then every $f \in \mathscr{P}^{1}(M)$ satisfies strong transversality.

In this paper the following two theorems will be proved.

Tileorem A. There exists a closed $C^{\infty} 3$-manifold $M$ such that set of all diffeomorphisms having POTP is not dense in $\operatorname{Diff}^{1}(M)$.

The Theorem answers to a problem stated in Morimoto [7].

THeOREM B. If $M$ is a closed $C^{\infty}$ 3-manifold, then $\mathscr{P}^{1}(M)$ is characterized as Axiom $A$ diffeomorphisms satisfying strong transversality.

A diffeomorphism $f$ of $M$ is quasi-Anosov if the fact that $\left\|D f^{n}(v)\right\|$ is bounded for all $n \in \mathbf{Z}$ implies that $v=0$. Theorem $\mathrm{A}$ is easily obtained in combining with Franks and Robinson [2] and Sakai [12]. The set of all quasi-Anosov diffeomorphisms belonging to $\operatorname{Diff}^{1}(M), \mathrm{QA}^{1}(M)$, is open and $\mathrm{QA}^{1}(M) \subset \mathscr{F}^{1}(M)$. It is easy to see that when $\operatorname{dim} M=2$, every $f \in \mathrm{QA}^{1}(M)$ is Anosov (see [5]). However an example of a diffeomorphism $f^{\prime}$ on the connected sum $M^{\prime}$ of two 3-tori that is quasi-Anosov but not Anosov was given in Franks and Robinson [2]. Since $f^{\prime}$ is $\Omega$-stable, there is $C^{1}$ neighborhood $\mathcal{U}$ of $f^{\prime}$ in $\operatorname{Diff}^{1}\left(M^{\prime}\right)$ such that every $g \in \mathcal{U}$ is quasi-Anosov but not Anosov. Thus, by [12] every $g \in \mathcal{U}$ cannot have POTP,

Received February 21, 1991. 
and so Theorem A is proved.

Before beginning the proof of Theorem $\mathrm{B}$ we give some notations and definitions.

Let $(X, d)$ be a compact metric space, and let $f: X \rightarrow X$ be a homeomorphism. A sequence of points $\left\{x_{i}\right\}_{i=a}^{b-1}(-\infty \leq a<b \leq \infty)$ in $X$ is called a $\delta$-pseudo orbit of $f$ if $d\left(f\left(x_{i}\right), x_{i+1}\right)<\delta$ for $a \leq i \leq b-1$. Given $\varepsilon>0$ a sequence of points $\left\{x_{i}\right\}_{i=a}^{b}$ is said to be $f-\varepsilon$-traced by a point $x \in X$ if $d\left(f^{i}(x), \mathrm{x}_{i}\right)<\varepsilon$ for $a \leq i \leq b$. We say that $f$ has the pseudo orbit tracing property (abbrev. POTP) if for $\varepsilon>0$ there is $\delta>0$ such that every $\delta$-pseudo orbit for $f$ can be $f-\varepsilon$-traced by some point of $X$. For compact spaces the notions stated above are independent of compatible metrics used. It is easy to see that if $f$ has POTP then the non-wandering set $\Omega(f)$ coincides with the chain recurrent set $R(f)$ for $f$, where $R(f)$ is the set of $x \in X$ such that for every $\delta>0$, there is a $\delta$-pseudo orbit of $f$ from $x$ to $x$ (see [11]). For $x \in X$ and $\varepsilon>0$ the local stable and unstable sets are defined by

$$
\begin{aligned}
& W_{\varepsilon}^{s}(y, f)=\left\{x \in X: d\left(f^{n}(x), f^{n}(y)\right) \leq \varepsilon \text { for all } n \geq 0\right\}, \\
& W_{\varepsilon}^{u}(x, f)=\left\{y \in X: d\left(f^{-n}(x), f^{-n}(y)\right) \leq \varepsilon \text { for all } n \geq 0\right\} .
\end{aligned}
$$

Suppose that $f$ has POTP. Then it is checked that for every $\varepsilon>0$, there is $0<\delta<\varepsilon / 2$ such that if $d(x, y)<\delta(x, y \in X)$ then

$$
W_{\varepsilon}^{s}(x, f) \cap W_{\varepsilon}^{u}(y, f) \neq \phi .
$$

Let $M$ be as before and denote by $d$ a Riemannian metric on $M$. Then for a hyperbolic set $\Lambda$ of $f \in \operatorname{Diff}^{1}(M)$ and for $x \in \Lambda$ the stable and unstable manifolds are defined by

$$
\begin{aligned}
& W^{s}(x, f)=\left\{y \in M: d\left(f^{n}(y), f^{n}(x)\right) \rightarrow 0 \text { as } n \rightarrow \infty\right\} . \\
& W^{u}(x, f)=\left\{y \in M: d\left(f^{-n}(y), f^{-n}(x)\right) \rightarrow 0 \text { as } n \rightarrow \infty\right\} .
\end{aligned}
$$

When $\Lambda$ can be written as the finite disjoint union $\Lambda=\Lambda_{1} \cup \cdots \cup \Lambda_{\ell}$ of closed invariant sets $\Lambda_{i}$ such that each of $f_{\mid \Lambda_{i}}$ is topologically transitive. Such a set $\Lambda_{1}$ is called a basic set with respect to $\Lambda$. The stable set $W^{s}\left(\Lambda_{i} . f\right)$ and unstable set $W^{u}\left(\Lambda_{i}, f\right)$ are defined by

$$
\begin{aligned}
& W^{s}\left(\Lambda_{i} . f\right)=\left\{y \in M: d\left(f^{n}(y), \Lambda_{i}\right) \rightarrow 0 \text { as } n \rightarrow \infty\right\} . \\
& W^{u}\left(\Lambda_{i} . f\right)=\left\{y \in M: d\left(f^{-n}(y), \Lambda_{i}\right) \rightarrow 0 \text { as } n \rightarrow \infty\right\} .
\end{aligned}
$$

Then $W^{\sigma}\left(\Lambda_{i}, f\right)=\bigcup\left\{W^{\sigma}(x, f): x \in \Lambda_{i}\right\}$ for $\sigma=s$, u. If $\varepsilon>0$ is small 
enough, then for $x \in \Lambda$ the local stable and unstable sets, $W_{\varepsilon}^{\sigma}(x, f)(\sigma=s, u)$, are $C^{1}$ disks tangent to certain subspaces $E^{s}(x)$ and $E^{u}(x)$, respectively, such that $x \mathrm{~T}_{x} M=E^{s}(x) \oplus E^{u}(x)$. Moreover there exists $0<\lambda<1$ such that

$$
\left\{\begin{array}{l}
d\left(f^{n}(y), f^{n}(z)\right) \leq \lambda^{n} d(y, z) \text { for } y, z \in W_{\varepsilon}^{s}(x, f) \text { and } n \geq 0 \\
d\left(f^{-n}(y), f^{-n}(z)\right) \leq \lambda^{n} d(y, z) \text { for } y, z \in W_{\varepsilon}^{u}(x, f) \text { and } n \geq 0
\end{array}\right.
$$

(see Hirsch and Pugh [3]). Thus $W_{\varepsilon}^{\sigma}(x, f) \subset W^{\sigma}(x, f)$ for $x \in \Lambda(\sigma=s, u)$ and

$$
\begin{aligned}
& W^{s}(x, f)=\bigcup_{n \geq 0} f^{-n}\left(W_{\varepsilon}^{s}\left(f^{n}(x), f\right)\right), \\
& W^{u}(x, f)=\bigcup_{n \geq 0}^{n} f^{n}\left(W_{\varepsilon}^{u}\left(f^{-n}(x), f\right)\right) .
\end{aligned}
$$

We denote $W^{\sigma}(x, f)$ by $W^{\sigma}(x)(\sigma=s, u)$ if there is no confusion.

If $f$ is Axiom $A$ diffeomorphism then we have $M=\cup\left(W^{\sigma}(x): x \in \Omega(f)\right\}$ for $\sigma=s, u$ and $\Omega(f)$ is expressed as the union $\Omega(f)=\Lambda_{1} \cup \cdots \cup \Lambda_{\ell}$ of disjoint basic sets for $f$. Such union is called the spectral decomposition for $f$. We say that $f$ has a cycle if there is a subsequence $\left\{\Lambda_{i j}\right\}_{j=1}^{s+1}(2 \leq s \leq \ell)$ of $\left\{\Lambda_{i}\right\}_{i=1}^{\ell}$ such that $W^{u}\left(\Lambda_{i j}\right) \cap W^{s}\left(\Lambda_{i j+1}\right) \neq \phi(1 \leq j \leq s)$ and $\Lambda_{i s+1}=\Lambda_{i 1}$. We say that $f$ satisfies the strong transversality condition if for all $x, y \in \Omega(f), W^{s}(x)$ and $W^{u}(y)$ meet transversely. Remark that $W^{s}\left(\Lambda_{i}\right) \cap W^{u}\left(\Lambda_{i}\right)=\Lambda_{i}$ for $1 \leq i \leq \ell$.

Now to obtain the conclusion of Theorem $\mathrm{B}$ it is enough to see that every $f \in$ $\mathscr{P}^{1}(M)$ satisfies strong transversality because $f$ satisfies Axiom $A$ as stated above.

Let $f \in \mathscr{P}^{1}(M)$ and $x \in M$. Then it was proved in [8] that if $0<\operatorname{dim}$ $W^{\sigma}(x)<\operatorname{dim} M$ for $\sigma=s, u$ then $T_{x} W^{s}(x) \not \subset T_{x} W^{u}(x)$ and $T_{x} W^{u}(x) \not \subset$ $T_{x} W^{s}(x)$. This tells us that if

$$
\operatorname{dim} W^{s}(x)+\operatorname{dim} W^{u}(x) \geq \operatorname{dim} M,
$$

then $W^{s}(x)$ and $W^{u}(x)$ meet transversely.

Therefore, to complete the proof of Theorem B it only remains to show (3) .

Since $f \in \mathscr{P}^{1}(M)$ satisfies Axiom $A, \Omega(f)$ is decomposed as $\Omega(f)=\Lambda_{1}$ $\cup \cdots \cup \Lambda_{\ell}$, where each $\Lambda_{i}$ is a basic set. Then by [4] for each $i$ there exists a compact neighborhood $B\left(\Lambda_{i}\right)$ satisfying the following (4), (5) and (6).

(4) There exists a continuous extension $T_{B\left(\Lambda_{i}\right)} M=\widetilde{E}_{i}^{s} \oplus \widetilde{E}_{i}^{u}$ of $T_{\Lambda_{i}} M=E_{i}^{s} \oplus E_{i}^{u}$ such that for $x \in B\left(\Lambda_{i}\right) \cap f^{-1}\left(B\left(\Lambda_{i}\right)\right)$,

$$
D_{x} f\left(\widetilde{E}_{i}^{s}(x)\right)=\widetilde{E}_{i}^{s}(f(x)) \text { and }\left\|D_{x} f_{\mid \tilde{E}_{i}^{s}(x)}\right\|<\lambda,
$$

and for $x \in B\left(\Lambda_{\imath}\right) \cap f\left(B\left(\Lambda_{i}\right)\right)$.

$$
D_{x} f^{-1}\left(\widetilde{E}_{i}^{u}(x)\right)=\widetilde{E}_{i}^{u}\left(f^{-1}(x)\right) \text { and }\left\|D_{x} f_{\mid \widetilde{E}_{i}^{u}(x)}^{-1}\right\|<\lambda .
$$


(5) There exists $\varepsilon_{1}>0$ such that for every $0<\varepsilon \leq \varepsilon_{1}$ there exist submanifolds $\widetilde{W}_{\varepsilon}^{\sigma}(x)\left(x \in B\left(\Lambda_{i}\right), \sigma=s, u\right)$ satisfying

(i) $f\left(\widetilde{W}_{\varepsilon}^{s}(x)\right) \subset \widetilde{W}_{\varepsilon}^{s}(f(x))$ and $d(f(x), f(y))<\lambda d(x, y)$ for evey $y \in \widetilde{W}_{\varepsilon}^{s}(x)$ if $x \in B\left(\Lambda_{i}\right) \cap f^{-1}\left(B\left(\Lambda_{i}\right)\right)$,

(ii) $f^{-1}\left(\widetilde{W}_{\varepsilon}^{u}(x)\right) \subset \widetilde{W}_{\varepsilon}^{u}\left(f^{-1}(x)\right)$ and $d\left(f^{-1}(x), f^{-1}(y)\right)<\lambda d(x, y)$ for evey $y \in \widetilde{W}_{\varepsilon}^{u}(x)$ if $x \in B\left(\Lambda_{i}\right) \cap f\left(B\left(\Lambda_{i}\right)\right)$.

(6) There exists $\delta>0$ such that if $d(x, y)<\delta\left(x, y \in B\left(\Lambda_{\imath}\right)\right)$ then $\widetilde{W}_{\varepsilon}^{\mathrm{s}}(x)$ and $\widetilde{W}_{\varepsilon}^{\mathrm{u}}(y)$ meet transversely.

For $E$ and $F$ subspaces of $T_{\Lambda_{i}} M$ define

$\tan \Varangle(F, E)=\sup \left\{\left\|\begin{array}{c}w_{2} \\ w_{1}\end{array}\right\|: w_{1} \in E, w_{2} \in E^{\perp}\right.$, and $\left.w_{1}+w_{2} \in F-\{0\}\right\}$.

Then we find $\theta_{1, i}>0$ satisfying $\tan \Varangle\left(E_{i}^{s}, E_{i}^{u \perp}\right)<\theta_{1, i}$ (See [10]). The continuity of $\widetilde{E}_{i}^{\sigma}(\sigma=s, u)$ ensures the existence of $\theta_{2, i}>0$ satisfying $\tan \Varangle\left(\widetilde{E}_{i}^{s}, \widetilde{E}_{i}^{u \perp}\right)$ $<\theta_{2, \text {. }}$.

Claim 1. Define $\theta_{2}=\max \left\{\theta_{2, i}: 1 \leq i \leq \ell\right\}$. For $0<\theta<\theta_{2}^{-1} \cdot\left(2+\theta_{2}\right)^{-1}$, there exists $K(\theta)>0$ such that $K(\theta) \rightarrow 0$ as $\theta \rightarrow 0$ and for every $v \in \mathrm{T}_{x} M(x \in$ $\left.B\left(\Lambda_{i}\right)\right)$ if $\tan \Varangle\left(v, \widetilde{E}_{i}^{u}(x)\right)<\theta$ and $\left\{x, f(x), \cdots, f^{N}(x)\right\} \subset B\left(\Lambda_{i}\right)$ for some $N>0$ then $\tan \Varangle\left(D_{x} f^{N}(v), \widetilde{E}_{i}^{u}\left(f^{N}(x)\right)\right) \leq K(\theta) \cdot \lambda^{2 N}$.

Proof. Let $x \in B\left(\Lambda_{i}\right)$ be fixed. For $v \in T_{x} M-\{0\}$, let $v=v^{s}+v^{u}=$ $(v)_{1}+(v)_{2}$, where $v^{s} \in \widetilde{E}_{i}^{s}, v^{u} \in \widetilde{E}_{i}^{u},(v)_{1} \in \widetilde{E}_{i}^{u}$, and $(v)_{2} \in \widetilde{E}_{i}^{u \perp}$. Clearly $\left(v^{s}\right)_{2}=$ $(v)_{2},\left(v^{s}\right)_{1}+v^{u}=(v)_{1},\left(v^{u}\right)_{1}=v^{u}$ and $\left(v^{u}\right)_{2}=0$. Since $f^{j}(x) \in B\left(\Lambda_{i}\right)$ for $0 \leq \mathrm{j}$ $\leq N$ and $\tan \Varangle\left(\widetilde{E}_{i}^{s}, \widetilde{E}_{i}^{u \perp}\right)<\theta_{2}$,

$$
\frac{\left\|\left(D_{x} f^{N}\left(v^{s}\right)\right)_{1}\right\|}{\left\|\left(D_{x} f^{N}(v)\right)_{2}\right\|}<\theta_{2}
$$

and since $f^{j}(x) \in B\left(\Lambda_{i}\right)$ for $0 \leq j \leq N$,

$$
\frac{\left\|D_{x} f^{N}\left(v^{u}\right)\right\|}{\left\|D_{x} f^{N}\left(v^{s}\right)\right\|} \geq \lambda^{-2 N} \frac{\left\|v^{u}\right\|}{\left\|v^{s}\right\|}
$$

It is checked that

$$
\begin{gathered}
\frac{\left\|v^{s}\right\|}{\left\|v^{u}\right\|} \leq \frac{\left(1+\theta_{2}\right)\left\|(v)_{2}\right\|}{\left\|(v)_{1}\right\|-\theta_{2}\left\|(v)_{2}\right\|}=\frac{1+\theta_{2}}{\left\|(v)_{1}\right\|-\theta_{2}} \leq \\
\quad \leq \frac{1+\theta_{2}}{\left\|(v)_{2}\right\|}=\frac{\theta\left(1+\theta_{2}\right)}{1-\theta+\theta_{2}} \text { and }
\end{gathered}
$$




$$
\frac{\left\|\left(D_{x} f^{N}(v)\right)_{2}\right\|}{\left\|\left(D_{x} f^{N}(v)\right)_{1}\right\|} \leq\left|\frac{\left\|\left(D_{x} f^{N}\left(v^{s}\right)\right)_{1}\right\|}{\left\|\left(D_{x} f^{N}\left(v^{s}\right)\right)_{2}\right\|}-\frac{\left\|\left(D_{x} f^{N}\left(v^{u}\right)\right)_{1}\right\|}{\left\|\left(D_{x} f^{N}\left(v^{s}\right)\right)_{2}\right\|}\right|^{-1} .
$$

From these inequalities we have

$$
\begin{gathered}
\left|\frac{\left\|\left(D_{x} f^{N}\left(v^{u}\right)\right)_{1}\right\|}{\left\|\left(D_{x} f^{N}\left(v^{s}\right)\right)_{2}\right\|}-\frac{\left\|\left(D_{x} f^{N}\left(v^{s}\right)\right)_{1}\right\|}{\left\|\left(D_{x} f^{N}\left(v^{s}\right)\right)_{2}\right\|}\right| \geq \lambda^{-2 N} \frac{\left\|v^{u}\right\|}{\left\|v^{s}\right\|}-\theta_{2} \geq \\
\geq \lambda^{-2 N} \frac{1-\theta \theta_{2}}{\theta\left(2+\theta_{2}\right)}-\theta_{2}=\lambda^{-2 N}\left(\frac{1-\theta \theta_{2}}{\theta\left(1+\theta_{2}\right)}-\lambda^{2 N} \cdot \theta_{2}\right),
\end{gathered}
$$

and so

$$
\frac{\left\|\left(D_{x} f^{N}(v)\right)_{2}\right\|}{\left\|\left(D_{x} f^{N}(v)\right)_{1}\right\|} \leq \lambda^{2 N} \cdot K(\theta)
$$

where $K(\theta)=\left(\frac{1-\theta \theta_{2}}{\theta\left(1+\theta^{2}\right)}-\theta_{2}\right)^{-1}$.

For $A$ a closed set of $M$, denote by $B_{r}(A)$ the closed neighborhood of $A$ with radius $r>0$.

Claim 2. Let $\Lambda_{i}$ and $\Lambda_{j}$ be the basic sets. Suppose that $2 \geq \operatorname{Ind} \Lambda_{j} \geq \operatorname{Ind} \Lambda_{j}$ $\geq 1$ where Ind $\Lambda$ denotes the dimension of the stable subbundle $E^{s}$ of a basic set $\Lambda$. Then there are $r_{1}>0\left(B_{r_{1}}\left(\Lambda_{i}\right) \subset B\left(\Lambda_{i}\right)\right.$ and $\theta>0$ such that if $x \in \Lambda$, and $y \in$ $W^{s}(x) \cap B_{r_{1}}\left(\Lambda_{i}\right)$, then $\tan \Varangle\left(T_{y} W^{s}(x), \widetilde{E}_{i}^{u}(y)\right)>\theta$.

Proof. If this is false, for every $\mathrm{n}>0$ there are $x_{n} \in \Lambda_{j}$ (Ind $\Lambda_{j} \geq$ Ind $\Lambda_{i}$ ) and $y_{n} \in W^{s}\left(x_{n}\right) \cap B_{1 / n}\left(\Lambda_{i}\right)$ such that $\tan \Varangle\left(T_{y_{n}} W^{s}\left(x_{n}\right), \widetilde{E}_{i}^{u}\left(y_{n}\right)\right)<1 / n$. Then, by (5) and (6) there are $z_{n} \in \Lambda_{i}$ and $w_{n}=\widetilde{W}_{\varepsilon_{1}}^{s}\left(y_{n}\right) \cap \widetilde{W}_{\varepsilon_{1}}^{u}\left(z_{n}\right)$. Since $y_{n} \rightarrow \Lambda_{i}$ as $n \rightarrow \infty$, there is a strictly increasing sequence $J_{n}>0$ such that $f^{k}\left(y_{n}\right) \in B\left(\Lambda_{i}\right)$ for $0 \leq k \leq J_{n}$ and $f^{J_{n}+1}\left(y_{n}\right) \notin B\left(\Lambda_{i}\right)$. Put $\tau=\inf \left\{d\left(x, \Lambda_{i}\right): x \in B\left(\Lambda_{i}\right)\right.$ and $\left.f^{-1}(x) \notin B\left(\Lambda_{\imath}\right)\right\}>0$. Since

$$
d\left(f^{j}\left(y_{n}\right), f^{j}\left(w_{n}\right)\right) \leq \lambda^{j} d\left(y_{n}, w_{n}\right) \text { for } 0 \leq j \leq J_{n},
$$

there is $N>0$ such that for every $n \geq N, f^{J n}\left(y_{n}\right) \in B\left(\Lambda_{i}\right) \backslash B_{\tau}\left(\Lambda_{i}\right)$ and $f^{J n}\left(w_{n}\right) \notin B_{\tau / 2}\left(\Lambda_{i}\right)$. Thus it is checked that there exists $c_{1}>0$ such that for every $n>0$,

$$
f^{\prime}\left(B_{c_{1}}\left(f^{J n}\left(y_{n}\right)\right)\right) \cap B_{c_{1}}\left(f^{J n}\left(y_{n}\right)\right)=\phi \text { for all } j>0 .
$$

Indeed, if for every $m>0$ there are $n_{m}>0, j_{m}>0$ and $x_{m}^{\prime} \in B_{\frac{1}{m}}\left(f^{J n_{m}}\left(y_{n_{m}}\right)\right)$ such that $f^{j m}\left(x_{m}^{\prime}\right) \in B_{\frac{1}{m}}\left(f^{J n_{m}}\left(y_{n_{m}}\right)\right)$, then $y=\lim _{m \rightarrow \infty} f^{J n_{m}}\left(y_{n m}\right) \in R(f)$, which is 
a contradiction since $y \notin \Omega(f)=R(f)$.

Similarly we can find $c_{2}>0$ such that for every $n>0$

$$
f^{j}\left(B_{c_{2}}\left(f^{J n}\left(w_{n}\right)\right)\right) \cap B_{c 2}\left(f^{J n}\left(w_{n}\right)\right)=\phi \text { for all } j>0 .
$$

Take and fix $0<\theta^{\prime}<\theta_{2}^{-1}\left(2+\theta_{2}\right)^{-1}$. Then there is $N^{\prime}>N$ such that for every $n \geq N^{\prime}, \tan \Varangle\left(T_{y_{n}} W^{s}\left(x_{n}\right), \widetilde{E}_{i}^{u}\left(y_{n}\right)\right) \leq \theta^{\prime}$.

By Claim 1, tan $\Varangle\left(D_{y_{n}} f^{\mathrm{J} n}\left(T_{y_{n}} W^{s}\left(x_{n}\right)\right), \widetilde{E}_{\imath}^{u}\left(f^{\mathrm{J} n}\left(y_{n}\right)\right)\right) \rightarrow 0$ as $n \rightarrow \infty$, and so

$$
\tan \Varangle\left(D_{y_{n}} f^{J n}\left(T_{y_{n}} W^{s}\left(x_{n}\right)\right), \mathscr{E}_{f n_{\left(y_{n}\right) \cdot f} J n_{\left(w_{n}\right)}}\left(\widetilde{E}_{i}^{u}\left(f^{\mathrm{Jn}}\left(w_{n}\right)\right)\right)\right) \rightarrow 0
$$

as $n \rightarrow \infty$ (by (7)). Here $\mathscr{E}_{x \circ y}$ denotes the parallel transform from $T_{x} M$ to $T_{y} M$. Then, from (7), (8) and (9) there are $n \geq N^{\prime}$ and $g \in \operatorname{Diff}^{1}(M)$ arbitrarily near to $f$ such that $W^{s}\left(x_{n}, g\right) \cap W^{u}\left(z_{n}, g\right) \neq \phi$ and $W^{s}\left(x_{n}, g\right)$ does not meet transversely to $W^{u}\left(z_{n}, g\right)$, thus contradiction since $g \in \mathscr{P}^{1}(M)$.

Claim 3 (Lemma IV. 8 of Mañé [6]). Let $E^{1}$ and $E^{2}$ be Banach spaces with norm $\|\cdot\|$, and denote by $B_{r}^{i}(p)$ the ball of radius $r$ in $E^{i}$ centered at $p$. Let $C>0$ and $\varepsilon^{\prime}>0$ be constants such that $\varepsilon^{\prime}$ is so small that $\varepsilon^{\prime} C<1$. For $\rho_{0}>0$ take $0<r$ $\leq \rho_{0}$ and $0<\varepsilon \leq \varepsilon^{\prime}$ satisfying

$$
\frac{\varepsilon\left(1+\varepsilon^{\prime}\right)}{1-\varepsilon^{\prime} C}<\frac{r-\varepsilon}{C} \text { and } \frac{\varepsilon\left(1+\varepsilon^{\prime}\right)}{1-\varepsilon^{\prime} C}<r .
$$

Suppose that $\phi: B_{\rho_{0}}^{1}(0) \rightarrow E^{2}$ and $\varphi: B_{r}^{2}(p) \rightarrow E^{1}$ are maps satisfying

(a) $\phi(0)=0,\left\|\phi\left(w_{1}\right)-\phi\left(w_{2}\right)\right\| \leq \varepsilon^{\prime}\left\|w_{1}-w_{2}\right\|$

$$
\text { for } w_{1}, w_{2} \in B_{\rho_{0}}^{1}(0) \text {, }
$$

(b) $\|\varphi(p)\|<\varepsilon,\left\|\varphi\left(w_{1}\right)-\varphi\left(w_{2}\right)\right\| \leq C\left\|w_{1}-w_{2}\right\|$

$$
\text { for } w_{1}, w_{2} \in B_{r}^{2}(p) \text {, and }
$$

(c) $\|p\|<\varepsilon$.

Then graph $\varphi \cap$ graph $\phi \neq \phi$, where graph $\phi=\left\{(w, \phi(w)): w \in B_{\rho_{0}}^{1}(0)\right\}$ and $\operatorname{graph} \varphi=\left\{(\varphi(w), w): w \in B_{r}^{2}(p)\right\}$. 


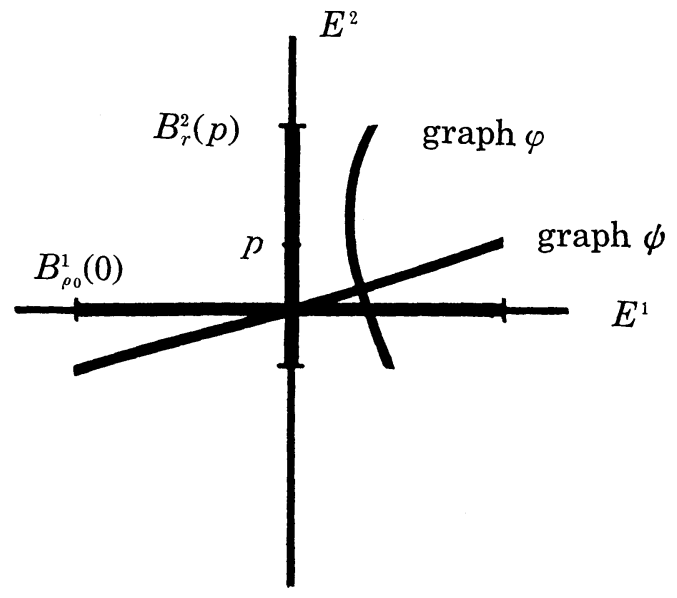

Firstly we show for every $0<\rho_{1}<\min \left\{r, \frac{1-\varepsilon}{C}\right\}, \varphi\left(B_{\rho_{1}}^{2}(p)\right) \subset B_{r}^{1}(0)$. Take and fix $y \in B_{\rho_{1}}^{2}(p)$. Since $\|y-p\|<\rho_{1}<\frac{r-1}{C}$, we have $C\|y-p\|+$ $\varepsilon<r$. Thus

$$
\|\varphi(y)\| \leq\|\varphi(\rho)\|+C\|y-p\| \leq \varepsilon+C\|y-p\|<r .
$$

From this a map $\phi \circ \varphi: B_{\rho_{1}}^{2}(p) \rightarrow E^{2}$ is well defined. Since $\|\varphi(\varphi(p))-\phi(0)\|$ $\leq \varepsilon^{\prime}\|\varphi(0)-0\|=\varepsilon^{\prime}\|\varphi(0)\|<\varepsilon^{\prime} \varepsilon$, we have $\|\phi(\varphi(p))\|<\varepsilon^{\prime} \varepsilon$ (by (a)). Therefore, for $w_{1}, w_{2} \in B_{\rho_{1}}^{2}(p)$

$$
\left\|\phi \varphi\left(w_{1}\right)-\phi \varphi\left(w_{2}\right)\right\| \leq \varepsilon^{\prime}\left\|\varphi\left(w_{1}\right)-\varphi\left(w_{2}\right)\right\| \leq \varepsilon^{\prime} C\left\|w_{1}-w_{2}\right\| ;
$$

i.e. $\phi \circ \varphi: B_{\rho_{1}}^{2}(p) \rightarrow E^{2}$ is contracting. If we choose $\frac{\varepsilon\left(1+\varepsilon^{\prime}\right)}{1+\varepsilon^{\prime} C}<\rho_{2}<\min \{r$, $\left.\frac{r-\varepsilon}{C}\right\}$, then for every $y \in B_{\rho_{2}}^{2}(p)$,

$$
\begin{aligned}
\|\phi \varphi(y)-p\| & \leq\|\phi \varphi(y)-\phi \varphi(p)\|+\|\phi \varphi(p)-p\| \leq \\
& \leq \varepsilon^{\prime} C\|y-p\|+\|\phi \varphi(p)\|+\|p\| \leq \\
& \leq \varepsilon^{\prime} C \rho_{2}+\varepsilon^{\prime} \varepsilon+\varepsilon<\rho_{2} .
\end{aligned}
$$

Thus $\psi^{\circ} \varphi: B_{\rho_{2}}^{2}(p) \rightarrow B_{\rho_{2}}^{2}(p)$ is a contraction. Thus there exists $z \in B_{\rho_{2}}^{2}(p)$ such that $\phi \cdot \varphi(z)=z$.

Theorem B will be proved under the above claims. The technique of the proof is to derive a contradiction in proving the existence of a cycle among basic sets $\Lambda_{1}, \cdots, \Lambda_{\ell}$ under the assumption that $f$ does not satisfy strong transversality. Remark that the dimension of $M$ is 3 . To prove Theorem B it is enough to see that 
for $x \in M \backslash \Omega(f), \operatorname{dim} W^{s}(x)+\operatorname{dim} W^{u}(x) \geq \operatorname{dim} M$ as explained before.

Suppose that there is $x \in M \backslash \Omega(f)$ such that $\operatorname{dim} W^{s}(x)+\operatorname{dim}$ $W^{u}(x)<\operatorname{dim} M=3$ (i.e. $\left.\operatorname{dim} W^{s}(x)=\operatorname{dim} W^{u}(x)=1\right)$. Since $f \in \mathscr{g}^{1}(M)$, there are $g \in \mathscr{p}^{1}(M)$ and $\alpha>0$ such that $f=g$ on a neighborhood of $\Omega(f)$, $\operatorname{dim}$ $W^{\sigma}(x, g)=\operatorname{dim} W^{\sigma}(x)$ for $\sigma=s, u$ and for the components $C^{\sigma}(x)$ of $x$ in $W^{\sigma}(x, g) \cap B_{\alpha}(x)(\sigma=s, u), C^{s}(x) \cap C^{u}(x)=\{x\}$.

Let $\Omega(g)=\Lambda_{1}(g) \cup \cdots \cup \Lambda_{\ell}(g)$ be a spectral decomposition for $g$. Then there are $1 \leq i \neq j \leq \ell, y \in \Lambda_{i}(g)$ and $z \in \Lambda_{j}(g)$ such that $W^{s}(x, g)=$ $W^{s}(z, g)$. and $W^{u}(x, g)=W^{u}(y, g)$. For simplicity suppose $y \in \Lambda_{1}(g)$ and $z \in \Lambda_{2}(g)$. Let $0<\varepsilon_{0}<r_{1} / 2$ be a number as in (2) and fix $N>0$ such that $g^{-N}(x) \in W_{\varepsilon_{0} / 2}^{u}\left(g^{-N}(y), g\right)$ and $g^{N}(x) \in W_{\varepsilon_{0} / 2}^{s}\left(g^{N}(z), g\right)$. Given the connected component $C_{g_{(x)}^{-N}}$ of $g^{-N}(x)$ in $B_{\varepsilon_{0} / 4}\left(g^{-N}(x)\right) \cap W_{\varepsilon_{0}}^{u}\left(g^{-N}(y), g\right)$, we have $C_{g_{(x)}^{-N}}$ $=\mathrm{B}_{\varepsilon_{0} / 4}\left(g^{-N}(x)\right) \cap W_{\varepsilon_{0}}^{u}\left(g^{-N}(y), g\right)$. Thus there is $0<\varepsilon \leq \varepsilon_{0} / 8$ such that $B_{\varepsilon}\left(g^{N}(x)\right) \cap g^{2 N}\left(C_{g_{(x)}^{-N}}\right)$ is the connected component of $g^{N}(x)$ in $B_{\varepsilon}\left(g^{N}(x)\right) \cap$ $g^{2 N}\left(C_{\varepsilon-N(x)}\right)$.

Denote by $C_{g^{N}(x)}$ the connected component of $g^{N}(x)$ in $B_{\varepsilon}\left(g^{N}(x)\right) \cap$ $g^{2 N}\left(C_{g^{-N(x)}}\right)$, and take and fix $0<\varepsilon_{2} \leq \varepsilon / 2$ such that $d(v, \mathrm{w})<\varepsilon_{2}(v, w \in M)$ implies $d\left(g^{-2 N}(v), g^{-N}(w)\right)<\varepsilon_{0} / 8$.

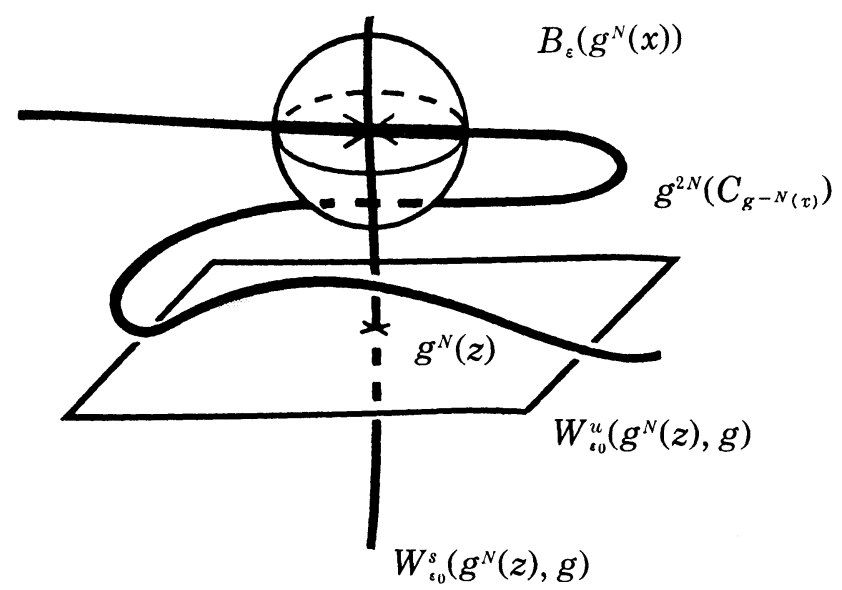

Claim 4. Fix any $w \in C_{g^{N}(x)} \cap B_{\varepsilon_{2}}\left(g^{N}(x)\right) \backslash\left\{g^{N}(x)\right\}$. If there exists $0<r^{\prime}$ $<\varepsilon_{2}$ such that $B_{r^{\prime}}(w) \cap C_{g^{N}(x)} \subset C_{g^{N}(x)} \backslash\left\{g^{N}(x)\right\}$, and if for every $w^{\prime} \in B_{r^{\prime}}(w) \cap$ $C_{g^{N}(x)}, \operatorname{dim} W^{s}\left(w^{\prime}, g\right)=1$, then $\operatorname{dim} W^{s}(v, \mathrm{~g})=1$ for every $v \in B_{\delta^{\prime}}(w) \backslash C_{g^{N}(x)}$. Here $0<\delta^{\prime}=\delta^{\prime}\left(\boldsymbol{r}^{\prime}, g\right)<\boldsymbol{r}^{\prime}$ is a number satisfying the property (1).

Proof. Note that if there is $v \in B_{\delta^{\prime}}(w) \backslash C_{g_{(x)}^{N}}$ such that $\operatorname{dim} W^{s}(v, g)=2$, then $W^{s}(v, g) \cap\left(B_{r^{\prime}}(w) \cap C_{g_{(x)}^{N}}=\phi\right.$. Since $w \in C_{g_{(x)}^{N}} \cap B_{\varepsilon_{2}}\left(g^{N}(x)\right)$, we have $g^{-2 N}(w) \in \mathrm{B}_{\varepsilon_{0} / 8}\left(g^{-N}(x)\right) \cap W_{\varepsilon_{0}}^{u}\left(g^{-N}(y), g\right)$ and hence 


$$
d\left(g^{-2 N-n}(w), g^{-N-n}(x)\right) \leq \varepsilon_{0} / 8 \text { for all } n \geq 0 .
$$

Since $d(v, w)<\delta^{\prime}$, by (1) there is $v^{\prime} \in M$ such that $d\left(g^{n}\left(v^{\prime}\right), g^{n}(v)\right)<r^{\prime}<\varepsilon_{0}$ for all $n \geq 0$ and $d\left(g^{-n}\left(v^{\prime}\right), g^{-n}(w)\right)<r^{\prime}<\varepsilon / 2<\varepsilon_{0} / 8$ for all $n \geq 0$. Thus

$$
v^{\prime} \in W^{s}(v, g) \text {. }
$$

By using (11) it is checked that $d\left(g^{-2 N-n}\left(v^{\prime}\right), g^{-N-n}(x)\right)<\varepsilon_{0} / 4$ for all $n$ $\geq 0$ (i.e. $\left.g^{-2 N}\left(v^{\prime}\right) \in C_{g^{-N}(x)}\right) \quad$ Thus $v^{\prime} \in g^{2 N}\left(C_{g^{-N}(x)}\right) \cap B_{\varepsilon}\left(g^{N}(x)\right)=C g_{(x)}$ (since $\left.d\left(v^{\prime}, g^{N}(x)\right) \leq d\left(v^{\prime}, w\right)+d\left(w, g^{N}(x)\right)<\varepsilon / 2+\varepsilon_{2}<\varepsilon\right)$. Since $d\left(v^{\prime}, w\right)$ $<r^{\prime}$, we have $v^{\prime} \in B_{r^{\prime}}(w) \cap C_{g_{(x)}^{N}}$. By (12)

$$
W^{2}(v, g) \cap\left(B_{r^{\prime}}(w) \cap C_{g_{(x)}}\right) \neq \phi
$$

which is a contradiction.

For $n \geq 1$ denote as $C_{g^{N+n}(x)}$ the connected component of $g^{N+n}(x)$ in $B_{\varepsilon}($ $\left.g^{N+n}(z)\right) \cap g\left(C_{g^{N+n-1}(x)}\right)$. Note that $C_{g^{N+n}(x)} \subset g\left(C_{g^{N+n-1}(x)}\right)$ for all $n \geq 1$.

Claim 5. For every $n>0$ and $0<\delta \leq \varepsilon_{0}$, there is $N^{\prime}>n$ such that for every $w \in B_{1 / N^{\prime}}\left(g^{N+N^{\prime}}(x)\right)$,

$$
C_{w} \cap W_{\delta / 2}^{u}\left(g^{N+N^{\prime}}(z), g\right) \neq \phi,
$$

where $C_{w}$ is the connected component of $w$ in $W^{s}(w, g) \cap B_{\varepsilon_{0}}(w)$.

Proof. If this is false, then there are $n_{0}>0, \delta_{0}>0$ and $w_{n} \in B_{1 / n}\left(g^{N+n}(x)\right)$ for all $n \geq n_{0}$ such that $C_{w_{n}} \cap W_{\zeta_{0}}^{u}\left(g^{N+n}(z), g\right)=\phi$. Let $r_{1}>0$ and $\theta>0$ be numbers given in Claim 2 for $g \in g^{1}(M)$. Clearly

$$
\begin{aligned}
d\left(w_{n}, g^{N+n}(z)\right) & \leq d\left(w_{n}, g^{N+n}(x)\right)+d\left(g^{N+n}(x), g^{N+n}(z)\right) \\
& \leq 1 / n+\lambda^{n} d\left(g^{N}(x), g^{N}(z)\right) \rightarrow 0
\end{aligned}
$$

as $n \rightarrow \infty$ by (2).

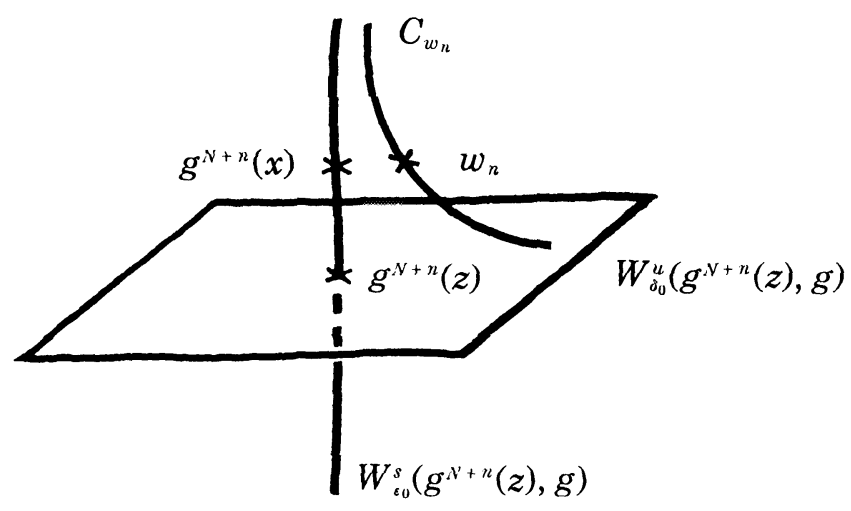


For a moment we treat a neighborhood of $g^{N+n}(z)$ as it were $\mathbf{R}^{3}$. Let $E_{n}^{1}=$ $T_{g^{N+n}(x)} W_{\delta 0}^{u}\left(g^{N+n}(z), g\right), E_{n}^{2}=T_{g^{N+n}(x)} W_{\varepsilon_{0}}^{s}\left(g^{N+n}(z), g\right)$ and fix $n_{1}>0$ such that $d\left(g^{N+n}(x), g^{N+n}(z)\right)<r_{1}$ for $n \geq n_{1}$. Remark that $g^{N+n}(z) \in \Lambda_{2}(g)$ and put $p_{n}$ $=\widetilde{E}_{2}^{u}\left(g^{N+n}(x)\right) \cap E_{n}^{2}$ for $n \geq n_{1}$. Then

$$
d\left(p_{n}, g^{N+n}(z)\right) \rightarrow 0 \text { as } n \rightarrow \infty .
$$

Thus, by Claim 2 there are constants $C=C(\theta)>0, r_{2}=r_{2}(\theta)>0$ and $n_{2} \geq n_{1}$ such that for every $n \geq n_{2}$ there is a map $\varphi_{n}: B_{r_{2}}^{2}\left(p_{n}\right) \rightarrow E_{n}^{1}$ satisfying

$(* *)$

$$
\left\|\varphi_{n}\left(p_{n}\right)\right\| \rightarrow 0 \text { as } n \rightarrow \infty \text { and }
$$

$$
\left\{\begin{array}{l}
\left\|\varphi_{n}\left(w_{1}^{\prime}\right)-\varphi_{n}\left(w_{2}^{\prime}\right)\right\| \leq C\left\|w_{1}^{\prime}-w_{2}^{\prime}\right\| \text { for } w_{1}^{\prime}, w_{2}^{\prime} \in B_{r_{2}}^{2}\left(p_{n}\right), \\
\text { graph } \varphi_{n} \subset C_{w n} .
\end{array}\right.
$$

Fix $0<\varepsilon^{\prime}<1$ such that $0<C \varepsilon^{\prime}<1$. Then there are $0<\rho_{0}<\delta_{0}$ and maps $\psi_{n}: B_{\rho_{0}}^{1}(0) \rightarrow E_{n}^{2}$ for $n>0$ such that

$$
\left\{\begin{array}{l}
\phi_{n}(0)=0, \\
\left\|\phi_{n}\left(w_{1}^{\prime}\right)-\phi_{n}\left(w_{2}^{\prime}\right)\right\| \leq \varepsilon\left\|w_{1}^{\prime}-w_{2}^{\prime}\right\| \text { for } w_{1}^{\prime}, w^{2} \in B_{\rho_{0}}^{1}(0) \text { and } \\
\text { graph } \phi_{n} \subset W_{\delta_{0}}^{u}\left(g^{N+n}(z), g\right) \text { for } n>0 .
\end{array}\right.
$$

Put $r=\min \left\{\rho_{0}, r_{2}\right\}$ and fix $0<\varepsilon \leq \varepsilon^{\prime}$ such that satisfies (10). Then, from $(*)$ and $(* *)$ we can take an integer $n_{3} \geq n_{2}$ such that for every $n \geq n_{3}, \phi_{n}$ and $\varphi_{n}$ satisfy the assumptions of Claim 3 . Thus

$$
C_{w_{n}} \cap W_{\delta_{0}}^{u}\left(g^{N+n}(z), g\right) \neq \phi .
$$

This is a contradiction.

Take and fix $n>0$ such that $\left.d\left(g^{N+n}(x)\right), g^{N+n}(z)\right)<\delta / 2$ where $0<\delta$ $=\delta(\varepsilon, g)<\varepsilon$ is a number given in (1). Let $N^{\prime}=N^{\prime}(n, \delta) \geq n$ be as in Claim 5 and put

$$
B_{\delta / 2}^{u}\left(g^{N+N^{\prime}}(z)\right)=B_{\delta / 2}\left(g^{N+N^{\prime}}(z)\right) \cap W_{\varepsilon_{0}}^{u}\left(g^{N+N^{\prime}}(z), g\right)
$$

Claim 6. There exists $w \in B_{\delta / 2}^{u}\left(g^{N+N^{\prime}}(z)\right) \backslash\left\{g^{N+N^{\prime}}(z)\right\}$ such that dim $W^{s}(w, g)=1$ and $C_{w} \cap C_{g^{N+n^{\prime}}(x)}=\phi$ where $C_{w}$ is the connected component of $w$ in $W^{s}(w, g) \cap B_{\varepsilon_{0}}\left(g^{N+N^{\prime}}(z)\right)$.

Before beginning with the proof of the Claim 6 we remark the following properties. 
Remarks. (i) For every tubular neighborhood $U$ of $W_{\varepsilon_{0}}^{s}\left(g^{N+N^{\prime}}(z), g\right)$, there is no sink periodic point $p$ of $g$ such that $U \backslash W_{\varepsilon_{0}}^{s}\left(g^{N+N^{\prime}}(z), g\right) \subset W^{s}(p, g)$.

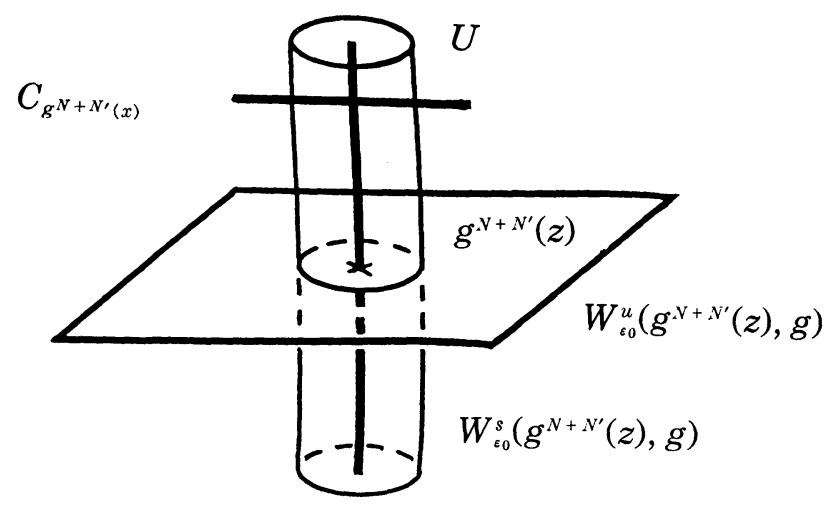

To prove this, we suppose that there is a sink $p$ satisfying $U \backslash W_{\varepsilon_{0}}^{s}($ $\left.g^{N+N^{\prime}}(z), g\right) \subset W^{s}(p, g)$. Then there are $N^{\prime \prime}>N^{\prime}$ and $0<\varepsilon^{\prime \prime} \leq \varepsilon$ such that for every $n \geq N^{\prime \prime}$ and $0<\hat{\varepsilon} \leq \varepsilon^{\prime \prime}, C_{g^{N+n}(x)} \cap B_{\hat{\varepsilon}}\left(g^{N+n}(z)\right) \subset U$. For $n \geq N^{\prime \prime}$ we put

$$
S^{u}\left(g^{N+n}(z)\right)=\partial B_{\varepsilon_{0}}^{u}\left(g^{N+n}(z)\right),
$$

and for $0<\hat{\varepsilon} \leq \varepsilon$ let $\hat{\delta}>0$ be a number as in the definition of POTP of $g$. Then for every $\hat{\delta}$ there are $n_{1}(\hat{\delta}), n_{2}(\hat{\delta}) \geq N^{\prime \prime}$ such that

$$
d\left(g^{N+n_{1}(\hat{\delta})}(x), \mathrm{g}^{N+n_{1}(\hat{\delta})}(z)\right)<\frac{\hat{\delta}}{2}
$$

and

$$
d\left(g^{N+n_{1}(\hat{\delta})}(z), w\right)<\frac{\hat{\delta}}{2}
$$

for every $w \in g^{-n_{2}(\hat{\delta})}\left(S^{\mathrm{u}}\left(g^{N+n_{1}(\hat{\delta})+n_{2}(\hat{\delta})}(z)\right)\right)$. Thus for every $w \in g^{-n_{2}(\hat{\delta})}\left(S^{\mathrm{u}}\right.$ $\left.\left(g^{N+n_{1}(\hat{\delta})+n_{2}(\hat{\delta})}(z)\right)\right)$,

$$
\left\{\cdots, g^{N+n_{1}(\hat{\delta})-2}(x), g^{N+n_{1}(\hat{\delta})-1}(x), w, g(w), \cdots\right\}
$$

is a $\hat{\delta}$-pseudo orbit for $g$. However it is easy to see that if we fix $\hat{\varepsilon}$ small enough, then there exists a $\hat{\delta}$-pseudo orbit among them which can not be $g-\hat{\varepsilon}$-traced since

$$
C_{g^{N+n_{1}(\hat{\delta})}(x)} \cap B_{\hat{\varepsilon}}\left(g^{N+n_{2}(\hat{\delta})}(x)\right) \subset U .
$$

(ii) There is no stable manifold $W^{s}(w, g)$ with $\operatorname{dim} W^{s}(w, g)=2$ and $\overline{W^{s}(w, g)} \supset W^{s}\left(g^{N+N^{\prime}}(z), g\right)$ such that there is a sequence of points $\left\{w_{n}\right\}$ in $W^{s}(w, g) \cap C_{g^{N+N^{\prime}}(x)} \backslash\left\{g^{N+N^{\prime}}(x)\right\}$ satisfying $w_{n} \rightarrow g^{N+N^{\prime}}(x)$ and $d_{s}\left(w_{n}, w_{n+1}\right) \rightarrow$ 
0 as $n \rightarrow \infty$. Here $d_{s}$ is a metric on $W^{s}(w, g)$ induced from $\|\cdot\|$.

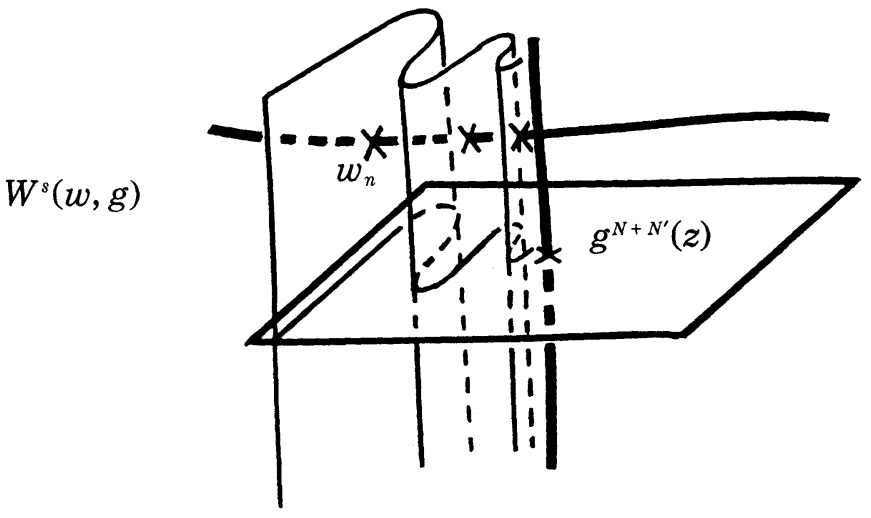

In fact, if there exists such a stable manifold then we can find $v \in$ $g^{-2 N-N^{\prime}}\left(W^{s}(w, g)\right)$ such that

$$
\tan \Varangle\left(T_{v} W^{s}\left(g^{-2 N+N^{\prime}}(w), g\right), \widetilde{E}_{1}^{u}(v)\right)<\theta,
$$

where $\theta>0$ is a number given in Claim 2 for $g$. This is absurd since $W^{s}(w, g)$ $\not \subset W^{s}\left(\Lambda_{1}(g), g\right)$.

(iii) If there is a sequence of points $\left\{w_{n}\right\}$ in $C_{g^{N+N^{\prime}}(x)}$ such that $w_{n} \rightarrow$ $g^{N+N^{\prime}}(x), \overline{\left(W^{s}\left(w_{n}, g\right)\right)} \rightarrow 0$ as $n \rightarrow \infty$ and $\operatorname{dim} W^{s}\left(w_{n}, g\right)=2$ for all $n \geq 0$, then Claim 6 is true. Here $r \overline{\left(W^{s}(w, g)\right)}$ denotes the maximal radius of a closed ball in $\overline{\left(W^{s}(w, g)\right)}$ centered at $w$ with respect to $d_{s}$.

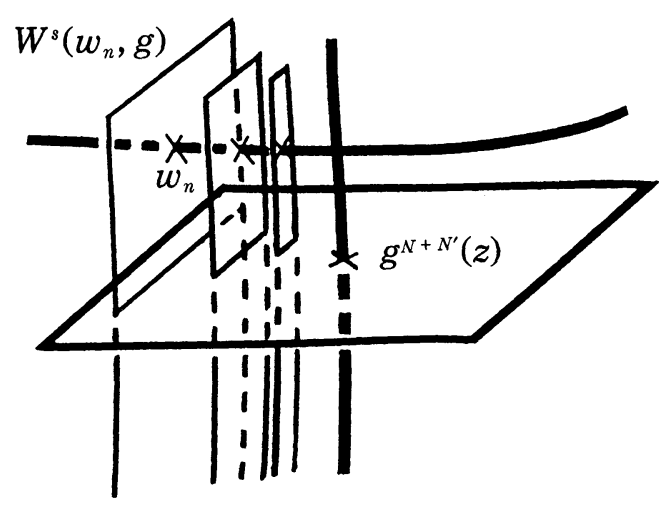

Indeed, fix $n>0$ and let $\theta>0$ be a number given in Claim 2 for $g$. Suppose that there is $v \notin W^{s}\left(w_{n}, g\right)$ such that $\operatorname{dim} W^{s}(v, g)=2$ and $\partial W^{s}\left(w_{n}, g\right)$ $\cap W^{s}(v, g) \neq \phi$. If we pick a point $v^{\prime} \in \partial W^{s}\left(w_{n}, g\right) \cap W^{s}(v, g)$, then there are sufficiently large $m>0$ and $v^{\prime \prime} \in W^{s}\left(g^{m}\left(w_{n}\right), g\right)$ arbitrarily near to $g^{m}\left(v^{\prime}\right)$ 
such that

$$
\tan \Varangle\left(T_{v^{\prime}} W^{s}\left(g^{m}\left(w_{n}\right), g\right), \widetilde{E}_{1}^{u}\left(v^{\prime \prime}\right)\right)<\theta .
$$

This is a contradiction. Thus $\partial W^{s}\left(w_{n}, g\right)$ consists of two 1-dimensional stable manifolds (since $U\left\{W^{s}(p, g): p\right.$ is a sink periodic point of $\left.g\right\}$ is open in $M$ ).

Proof of Claim 6. We divides the proof into two cases.

Case 1. For every

$$
w \in C_{g^{N+N^{\prime}}(x)} \cap B_{1 / 2 N^{\prime}}\left(g^{N+N^{\prime}}(z)\right\} \backslash\left\{\mathrm{g}^{N+N^{\prime}}(z)\right\}
$$

and $0<r^{\prime}<1 / N^{\prime}$ such that

$$
B_{r^{\prime}}(w) \cap C_{g^{N+N^{\prime}}(x)} \subset C_{g^{N+N^{\prime}}(x)} \backslash\left\{g^{N+N^{\prime}}(x)\right\} .
$$

there is $w^{\prime} \in B_{r^{\prime}}(w) \cap C_{g^{N+N^{\prime}}(x)}$ such that $\operatorname{dim} W^{s}\left(w^{\prime}, g\right) \geq 2$.

Note that $\bigcup\left\{W^{s}\left(\Lambda_{k}(g), g\right): \Lambda_{k}(g)\right.$ is an attractor $\}$ is an open set of $M$. By Remarks (i), (ii) and (iii) we may assume that there is $w \in W_{\delta / 2}^{u}\left(g^{N+N^{\prime}}(z), g\right) \backslash$ $\left\{g^{N+N^{\prime}}(z)\right\}$ such that $\operatorname{dim} W^{s}(w, g)=2, \overline{C_{w}} \cap W^{s}\left(g^{N+N^{\prime}}(z), g\right) \neq \phi$ and $C_{w} \cap$ $C_{g^{N+N^{\prime}}(x)}=\phi$. Here $C_{w}$ denotes the connected component of $w$ in $W^{s}(w, g) \cap$ $B_{\varepsilon_{0}}\left(g^{N+N^{\prime}}(z)\right)$.

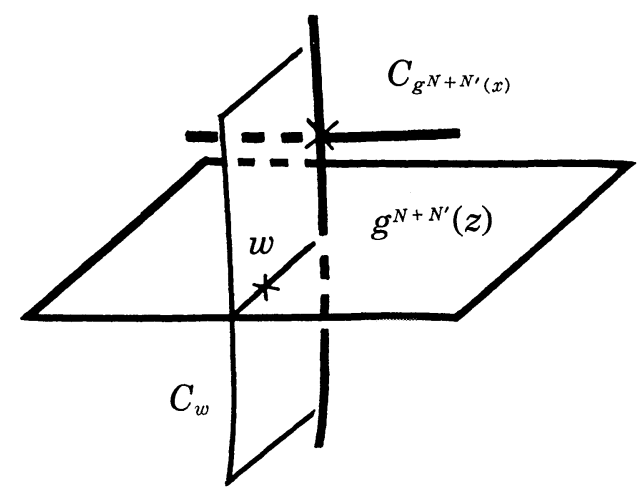

For every $0<\beta \leq \varepsilon$, let $0<\gamma(\beta) \leq \beta$ be a number as in the definition of POTP of $g$. Take $v \in B_{r(\beta)}\left(g^{N+N^{\prime}}(x)\right) \cap C_{w}$. Then

$$
\left\{\cdots, g^{N+N^{\prime}-2}(x), g^{N+N^{\prime}-1}(x), v, g(v), \cdots\right\}
$$

is a $\gamma(\beta)$-pseudo orbit of $g$. Thus there exists $\hat{v} \in C_{g^{N+N^{\prime}}(x)}$ such that $d\left(g^{n}(v)\right.$, $\left.g^{n}(\hat{v})\right)<\beta$ for all $n \in \mathbf{Z}$, On the other hand, since $v \notin W^{s}\left(\Lambda_{2}(g), g\right)$, there exists $n_{v, \beta}>0$, such that $g^{i}(v) \in B_{r_{1} / 2}\left(\Lambda_{2}(g)\right)$ for $0 \leq \mathrm{i} \leq n_{v, \beta}$ and $g^{n_{v, \beta}+1}(v) \notin$ $\mathrm{B}_{r_{1 / 2}}\left(\Lambda_{2}(\mathrm{~g})\right)$. Thus we have $B_{\varepsilon}\left(g^{i}(v)\right) \subset B_{r_{1}}\left(\Lambda_{2}(g)\right)$ for $0 \leq i \leq n_{v, \beta}$. Let be $C_{\hat{v}}$ be the connected component of $\hat{v}$ in $C_{g^{N+N^{\prime}}(x)} \cap B_{\varepsilon}(\hat{v})$. Then, by the hyperbolicity 
of $B_{r_{1}}\left(\Lambda_{2}(g)\right)$ there is $0<\varepsilon_{3} \leq \varepsilon$ such that

$$
\inf _{v, \beta} d_{u}\left(\partial\left(g^{n}{ }^{v, \beta}\left(C_{\hat{v}}\right)\right) \cdot g^{n}{ }^{v, \beta}(\hat{v})\right) \geq \varepsilon_{3},
$$

where $d_{u}$ denotes a metric on unstable manifolds induced from $\|\cdot\|$. Since $g \in \mathscr{P}^{1}(M)$ and $g^{i}\left(C_{\hat{v}}\right) \cap g^{i}\left(C_{\omega}\right)=\phi$ for $0 \leq i \leq n_{v, \beta}$, by using the methods stated in the proofs of Claims 2 and 4 we can find $g^{\prime} \in \operatorname{Diff}^{1}(M)$ arbitrarily near to $g$ such that $\left.g^{\prime}\right|_{\Omega(g)}=\left.g\right|_{\Omega(g)}, W^{s}\left(g^{n}{ }^{v, \beta}(v), g^{\prime}\right) \cap W^{u}\left(g^{n, \beta}(\hat{v}), g^{\prime}\right) \neq \phi$, and $W^{2}\left(g^{n, \beta}(v), g^{\prime}\right)$ does not meet transversely to $W^{u}\left(g^{n, \beta}(\hat{v}), g^{\prime}\right)$. This is a contradiction.

Case 2. There exists

$$
w \in C_{g^{N+N^{\prime}}(x)} \cap B_{1 / 2 N^{\prime}}\left(g^{N+N^{\prime}}(\mathrm{x})\right) \backslash\left\{g^{N+N^{\prime}}(x)\right\}
$$

and $0<r^{\prime}<1 / 2 N^{\prime}$ such that

$$
B_{r^{\prime}}(w) \cap C_{g^{N+N^{\prime}}(x)} \subset C_{g^{N+N^{\prime}}(x)} \backslash\left\{g^{N+N^{\prime}}(x)\right\}
$$

and for every $w^{\prime} \in B_{r^{\prime}}(w) \cap C_{g^{N+N^{\prime}}(x)}, \operatorname{dim} W^{s}\left(w^{\prime}, g\right)=1$.

By Claim 4, there is $0<\delta^{\prime}=\delta^{\prime}\left(r^{\prime}, g\right)<1 / 2 N^{\prime}$. such that for every $v \in$ $B_{\delta^{\prime}}(w) \backslash C_{g^{N+N^{\prime}}(x)}, \operatorname{dim} W^{s}(v, g)=1$. Denote by $C_{v}$ the connected component of $v$ in $W^{s}(v, g) \cap B_{\varepsilon_{0}}(v)$ for $v \in B_{\delta^{\prime}}(w) \backslash C_{g^{N+N^{*}}(x)}$. Take and fix $v \in B_{\delta^{\prime}}(w) \backslash$ $C_{g^{N+N^{\prime}}(x)}$ such that $C_{v} \cap C_{g^{N+N^{\prime}}(x)}=\phi$. Then there is $v^{\prime}=C_{v} \cap W_{\delta / 2}^{u}\left(g^{N+N^{\prime}}(z)\right.$, $g$ ) $\neq \phi$ by Claim 5 (since $v \in B_{1 / 2 N^{\prime}}\left(g^{N+N^{\prime}}(x)\right)$ ). This completes the proof of Claim 6.

It is checked that for every $w \in B_{\delta / 2}^{u}\left(g^{N+N^{\prime}}(z)\right)$,

$$
W^{s}(w, g) \cap C_{g^{N+N^{\prime}}(z)} \neq \phi .
$$

Indeed, since $d\left(w, g^{N+N^{\prime}}(x)\right)<\delta$ for $w \in B_{\delta / 2}^{u}\left(g^{N+N^{\prime}}(z)\right)$, there is $w^{\prime} \in M$ such that

$$
d\left(g^{n}\left(w^{\prime}\right), g^{n}(w)\right)<\varepsilon \text { for all } n \geq 0
$$

and

$$
d\left(g^{-n}\left(w^{\prime}\right), g^{N+N^{\prime}-n}(x)\right)<\varepsilon \text { for all } n \geq 0
$$

Thus $g^{-2 N+N^{\prime}}\left(w^{\prime}\right) \in C_{g_{(x)}^{-N}}$ and so $\mathrm{g}^{-N^{\prime}}\left(w^{\prime}\right) \in g^{2 N}\left(C_{g_{(x)}^{-N}}\right)$. Since $\mathrm{g}^{-N^{\prime}}\left(w^{\prime}\right) \in$ $B_{\varepsilon}\left(g^{N}(x)\right)$ (by (13)), we have $\mathrm{g}^{-N^{\prime}}\left(w^{\prime}\right) \in C_{g_{(x)}^{N}}$ and hence $w^{\prime} \in C_{g^{N+N^{\prime}}(x)}$. Thus $W^{s}(w, g) \cap C_{g^{N+N^{\prime}}(x)} \neq \phi$ since $w^{\prime} \in W^{s}(w, g)$.

Let $W \in B_{\delta / 2}^{u}\left(g^{N+N^{\prime}}(z)\right) \backslash\left\{g^{N+N^{\prime}}(z)\right\}$ be as in Claim 6. Then $w \in$ $W^{u}\left(\Lambda_{i}(g), g\right)$ and $\operatorname{dim} W^{s}(w, g)=1$. Since $M=\bigcup_{i=1}^{\ell} W^{s}\left(\Lambda_{i}(g), g\right)$, we may 
suppose that $w \in W^{s}\left(\Lambda_{3}(g), g\right)$. Clearly Ind $\Lambda_{3}(g)=1$ and

$$
w \in W^{u}\left(\Lambda_{2}(g), g\right) \cap W^{s}\left(\Lambda_{3}(g), g\right) \neq \phi .
$$

It is easy to see that $\Lambda_{2}(g) \neq \Lambda_{3}(g)$. For, if $\Lambda_{2}(g)=\Lambda_{3}(g)$ then $w \in$ $W^{u}\left(\Lambda_{2}(g), g\right) \cap W^{s}\left(\Lambda_{2}(g), g\right)=\Lambda_{2}(g)$. Thus $C_{g_{(x)}^{N+N^{\prime}}} \cap W_{\varepsilon}^{s}(w, g)=\phi$. However, since

$$
\left\{\cdots, g^{-1}(x), x, g(x), \cdots, g^{N+N^{\prime}-1}(z), w, g(w), \cdots\right\}
$$

is a $\delta$-pseudo orbit of $g$, we have $C_{g^{N+N^{\prime}}(x)} \cap W_{\varepsilon}^{s}(w, g)=\phi$. This is a contradiction. Hence $\Lambda_{2}(g) \neq \Lambda_{3}(g)$.

Since $w \in B_{\tilde{\delta} / 2}^{u}\left(g^{N+N^{\prime}}(z)\right)$, we have $W^{s}(w, g) \cap C_{g_{(x)}^{N+N^{\prime}}} \neq \phi$. Thus $W^{s}\left(\Lambda_{1}\right.$ $(g), g) \cap W^{u}\left(\Lambda_{3}(g), g\right) \neq \phi$.

The conclusions obtained above is summarized as follows

$$
\left\{\begin{array}{l}
\text { Ind } \Lambda_{3}(g)=1 \\
\Lambda_{2}(g) \neq \Lambda_{3}(g) \\
W^{\mathrm{u}}\left(\Lambda_{2}(g), g\right) \cap W^{\mathrm{s}}\left(\Lambda_{3}(g), g\right) \neq \phi \text { and } \\
W^{\mathrm{u}}\left(\Lambda_{1}(g), g\right) \cap W^{\mathrm{s}}\left(\Lambda_{3}(g), g\right) \neq \phi
\end{array}\right.
$$

By (14) there exists a cycle among basic sets of $g$. Indeed, since there are $z_{1} \in$ $\Lambda_{1}(g)$ and $z_{2} \in \Lambda_{2}(g)$ such that $W^{\mathrm{u}}\left(z_{1}, g\right) \cap W^{\mathrm{s}}\left(z_{2}, g\right) \neq \phi$ and $\operatorname{dim} W^{u}\left(z_{1}\right.$, $g)=\operatorname{dim} W^{s}\left(z_{2}, g\right)=1$, by (14) we can find $z_{3} \in \Lambda_{3}(g) \neq \Lambda_{2}(g)$ such that $W^{\mathrm{u}}\left(z_{1}, g\right) \cap W^{\mathrm{s}}\left(z_{3}, g\right) \neq \phi, \quad \operatorname{dim} \quad W^{s}\left(z_{3}, g\right)=1 \quad$ and $\quad W^{\mathrm{u}}\left(\Lambda_{2}(g), g\right) \cap$ $W^{\mathrm{s}}\left(\Lambda_{3}(g), g\right) \neq \phi$. Since $W^{\mathrm{u}}\left(z_{1}, g\right) \cap W^{\mathrm{s}}\left(z_{3}, g\right) \neq \phi$ and $\operatorname{dim} W^{u}\left(z_{1}, g\right)=$ $\operatorname{dim} W^{s}\left(z_{3}, g\right)=1$, by the same manner we can find $z_{4} \in \Lambda_{4}(g) \neq \Lambda_{3}(g)$ such that $W^{\mathrm{u}}\left(z_{1}, g\right) \cap W^{\mathrm{s}}\left(z_{4}, g\right) \neq \phi, \operatorname{dim} W^{s}\left(z_{4}, g\right)=1$ and $W^{\mathrm{u}}\left(\Lambda_{3}(g), g\right) \cap$ $W^{\mathrm{s}}\left(\Lambda_{4}(g), g\right) \neq \phi$. In this repetition we have a cycle among basic sets $\Lambda_{1}(g)$, $\cdots, \Lambda_{\ell}(g)$ and reach a contradiction. We finish the proof of Theorem B. 


\section{REFERENCES}

[1] N. Aoki, The set of Axiom $A$ diffeomorphisms with no cycle, to appear in Bol. Soc. Bras. Math.

[2] J. Franks and C. Robinson, A quasi-Anosov diffeomorphism that is not Anosov, Trans. AMS., 223 (1976), 267-278.

[3] M. Hirsch and C. Pugh, Stable manifolds and hyperbolic sets, in Global Analysis, Proc. Sympos. Pure Math. AMS., 14 (1970) , 133-163.

[4] M. Hirsch, J. Palis, C. Pugh and M. Shub, Neighborhoods of hyperbolic sets, Invent. Math., 9 (1970) , 121-134.

[5] R. Mañé, Quasi-Anosov diffeomorphisms and hyperbolic manifolds, Trans. AMS., 229 (1977), 351-370.

16] - . A proof of the $C^{1}$ stability conjecture, Publ. Math. IHES., 66 (1987), $161-210$.

17] A. Morimoto, The method of pseudo orbit tracing property and stability, Tokyo Univ. Seminary Notes 39, 1979. (In Japanese.)

[8] K. Moriyasu, Topological stability of diffeomorphisms, Nagoya Math J., 123 (1991), $91-102$.

19] J. Palis, On the $C^{1} \Omega$-stability conjecture, Publ. Math. IHES, 66 (1987), 211-215.

[10] F. Przytycki, Anosov endomorphisms, Studia Math., 58 (1976) . 249-283.

[11] C. Robinson, Stability theorem and hyperbolicity in dynamical systems, Rocky Mountain J. Math., 7 (1977), 425-437.

[12] K. Sakai, Quasi-Anosov diffemorphisms and pseudo orbit tracing property, Nagoya Math. J., 111 (1988), 111-114.

Kisarazu National College of Technology

Kisarazu, Chiba 2.92

Japan 